\title{
Eliminating the Uncertainty of the Concept of Energy
}

\author{
Etkin Valery Abramovich \\ Research Center, Toglyatti State University, Toglyatti, Russian Federation
}

Email address:

etkin.v@mail.ru

\section{To cite this article:}

Etkin Valery Abramovich. Eliminating the Uncertainty of the Concept of Energy. International Journal of Energy and Power Engineering. Vol. 8, No. 3, 2019, pp. 35-44. doi: 10.11648/j.ijepe.20190803.12

Received: June 11, 2019; Accepted: July 11, 2019; Published: August 8, 2019

\begin{abstract}
The article analyzes the reasons that led to the loss of the original meaning of energy as a quantitative measure of motion and the uncertainty of this concept. A generalization of the energy conservation law was proposed taking into account the kinetics and irreversibility of real processes, and on this basis, the general properties of any forms of energy were identified. The ability is shown to return energy close to the original meaning as the most common characteristic function of the system state, expressing its ability to perform any work. A new classification of forms of energy is given, based on the fundamental difference between ordered and disordered works as quantitative measures of the processes of its transfer and transformation, as well as the presence of an irreducible part in each form of energy.
\end{abstract}

Keywords: Energy, It's Meaning, Properties, Classification, Conservation Laws

\section{Introduction}

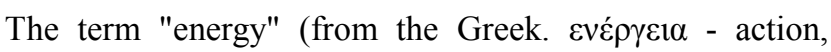
activity, strength, power) was proposed by the authoritative physicist T. Jung (1807) instead of the concept of "living force" $M v^{2}$ by authoritative physicist T. Jung (1807) [1]. It was adopted universally as a step towards further concretization of the very anthropomorphic concept of "action" introduced by Leibniz (1669). Leibniz defined it as the product $M v^{2}$ for the duration of the action $t$, or as the production of the amount of movement $m v$ on the traversed path $s$. Thus, initially, the energy was understood as a quantitative measure of movement. The introduction of the concept of force was also associated with movement (I. Newton, 1687). However, in the mid-19th century, the wellknown Scottish mechanic W. Renkin introduced the concept of "potential energy" $E^{p}$ as a quantitative measure of interaction. The potential energy was determined by the amount of work $W^{e}$ spent on the transfer of the system of bodies (or material points) from the initial configuration to this one, i.e. it was a function of their relative position. This required the introduction of another term "kinetic energy" $E^{k}$ (W. Thomson) as the antipode of "living force" [1].

Subsequently, the division of energy into potential and kinetic was fixed due to the proof by R. Clausius (1870) of the "virial theorem". According to it, the average kinetic energy of a set of mutually moving particles of a stable closed system is equal and opposite in sign to half of its potential energy if the forces connecting these particles are proportional to the square of the deviation of their coordinates from the center of mass of the system [1]. At the same time, the potential energy in accordance with the traditional interpretation of the law of Newton had a negative value, which left an imprint on all the subsequent development of classical and quantum mechanics.

Both quantities, $E^{p}$ and $E^{k}$, were considered in this case as relative, depending on the reference system (RS). This situation suited physicists quite well until it became impossible to neglect the irreversible phenomena associated with the transformation of mechanical forms of energy into heat energy. As a measure of thermal energy, the founder of thermodynamics R. Clausius proposed the concept of "total body heat", by which he understood the sum of heat $Q$, summed to the body from the outside during heat exchange, and the "work of disgregation" $W^{d}$, accompanied by heat generation inside the system. However, after a series of discussions, preference was given to the term "internal energy" $U$, proposed by W. Thomson [1]. From the standpoint of mechanics, the internal energy had the meaning of the "dispersed" (out of working capacity) part of the total energy of system $\mathcal{E}$, which ceased to depend on the movement of the system and its position relative to other bodies. Thus, the scope of the law of conservation of the 
amount of $E=E^{k}+E^{p}$ had to be limited to the so-called "conservative" (nondissipative) systems, and the law of conservation was attributed to "total" energy, which includes the internal energy $U$ :

$$
\mathcal{E}_{\mathrm{iz}}=\left(E^{\mathrm{K}}+E^{\mathrm{\Pi}}+U\right)_{\mathrm{iz}}=\text { const. }
$$

It would not be an exaggeration to say that it was the use of the term "energy" (albeit with the addition of "potential") to a quantity unrelated to movement, and the term "internal energy" to a value not measured by work that gave rise to difficulties in defining the concept of energy. Both potential and kinetic energy belonged to the whole set of interacting (mutually moving) bodies, that is, it was "mutual" (relative), depending on the reference system (RS). Such energy, by virtue of the conservation laws, did not belong to any of the bodies of a closed system separately, since a change in its position or movement due to conservation laws was accompanied by a change in the relative position or motion of all other bodies of the closed system. This required consideration as a research object of a closed (isolated) system, to which the conservation law (1) applied. However, for convenience, they began to attribute it to one body, as if in a "force field" of more massive "field-forming" bodies. The area of justice of such an approximation was limited to the so-called "test" bodies (and subsequently charges), so small that their introduction into the external "field" or movement in it did not introduce noticeable distortions into it. This could not be done with respect to macrosystems, whose internal energy $U$, unlike external $E$, by definition did not depend on the position and movement of the system relative to the external environment and any (inertial or noninertial) reference systems in it. Therefore, all its components could be measured only in absolute scales (temperatures, pressures, etc.), which remain unchanged in any processes in the system. Otherwise, obviously, the internal energy would also become dependent on RS, changing in the absence of heat exchange $d Q$ and $d W^{1)}$ in violation of the first law of thermodynamics as the law of conservation of internal energy [2]:

$$
d U=d Q-d W
$$

It would seem that this law deliberately excludes the internal energy $U$ from the sphere of competence of the special and general theory of relativity. However, they still do not stop trying to make heat, work and internal energy dependent on speed [2]. This deprives the value of $U$, which was originally embedded in it. Later it became clear that in the case of non-isolated (open-loop) systems, the division of energy into "external" and "internal" is not always possible. In particular, for polarized bodies, a part of the internal energy is dependent on the external field, and the energy of the field depends on their internal state (for example, temperature $T$ ). The situation became even more complicated

\footnotetext{
1) The sign of incomplete differential " $\bar{d}$ " in the expressions of elementary heat exchange $d Q$ and elementary work $d W$ emphasizes their dependence on the process path.
}

when it turned out that there was no isolation from gravitational fields or neutino flows. In this case, in accordance with the conservation law (2), the concept of an isolated system should include both gravitational and neutrino fields. This is only the Universe as a whole, which includes "all things", for which all its energy $\mathcal{E}$ out is "internal".

All this turns the energy conservation law (1) into some abstract "formula for calculating certain numerical values" [3], and the energy itself into "one of seven integrals of motion" [4]. In such a situation, as rightly pointed out by A. Poincare, we cannot say about energy "nothing beyond the fact that there is something that remains unchanged" [5]. For a physical quantity that changes (quantitatively or qualitatively) in the course of any process, tying together all the phenomena of nature, such uncertainty is completely unacceptable, especially since along with energy in isolated systems, such quantities as mass, charge, momentum and his moment.

The modern reader is often surprised, not finding in the reference books and encyclopedias a physically more meaningful definition of the concept of energy than the philosophical interpretation of it as "a general quantitative measure of movement and interaction of all types of matter" [6]. The interpretation of energy as a measure of interaction only increased the ambiguity of this concept, especially after quantum physics in its "standard model" introduced the concept of "exchange interaction" and practically excluded the concept of force and work from consideration. As a result, the energy (especially negative) finally ceased to be a measure of the efficiency of the system. Hence the recognition of R. Feynman that "the physics of today do not know what energy is" [7].

Under these conditions, it is advisable to try to at least partially return the concept of energy to its simple and clear original meaning of the quantitative measure of motion. The path proposed here is based on a logical-mathematical analysis, leading to the understanding of energy as the most general characteristic function of a state, the derivatives of which determine all other properties of the system by independent arguments. In the implementation of this task, we will proceed from the already proven and proven practice of the principles of energy dynamics as a theory, which sets as its main task the study of the most general laws of the processes of transfer and transformation of any form of energy [8]. Being a general physical discipline, it adheres to the deductive method of research (from general to particular) characteristic of classical thermodynamics and the phenomenological approach, that is, it avoids using hypotheses, postulates, considerations of statisticalmechanical nature and changing model ideas about the object of research in its foundations. At the same time, its characteristic cause-and-effect analysis, taking into account the irreversibility of any real processes, makes it possible to identify the most common and reliable properties of energy. 


\section{Oscillation of the Density of Primary Matter as a Single Source of Energy}

The concept of "primary matter" as the building material of "all things" goes back to the ideas of ancient civilizations and is reflected in the philosophies of the distant past. The presence of such a "primary" matter was confirmed in the recent discoveries of astronomers. They found that at least $95 \%$ of the mass of the entire Universe is "hidden", not participating in electromagnetic interactions and therefore "invisible" (unobservable) [9]. Recognition of this fact eliminates the negative effects of the expulsion from ether physics, which led to the substitution of even less defined concepts of "physical vacuum", "thin fields", "dark" matter and energy, "quintessence", etc.

The fact that primary matter becomes observable only as it is structured ("condensation") means that modern science has studied only a small, condensed ("baryon") part of the matter of the Universe, beginning from atoms and molecules, and ending with clusters of galaxies. The main (uncondensed, non-barion) part of the matter still remains outside the sphere of natural science. Meanwhile, without it, it is difficult to understand the nature of the concept of energy. Therefore, the primary object of consideration in this article is precisely the primary, unstructured (continual) matter, which we will not a priori associated with any particle models of the space environment.

We will consider the "primary" matter as a continuous pervasive medium, the condensation products of which occupy the same space. Possible discreteness of this medium does not prevent its mathematical description, if we use the concept of energy density $\rho_{e}(\mathrm{r}, t)=\partial \mathcal{E} / \partial V$ and matter $\rho(\mathrm{r}, t)=$ $\partial M / \partial V$ as functions of spatial coordinates (radius vector $r$ ) and time $t$. This eliminates the need for a preliminary solution to the problem of finite or infinite divisibility ("nesting") of matter, as well as the development of corpuscular models of this medium, which is hampered by the ever-expanding number of "subatomic" particles. Such an approach is all the more important because these models cannot be verified by modern methods of experimental physics.

For the continuum, the division of energy $\mathcal{E}$ into external $E$ and internal $U$ loses all meaning, since the concept of an external (surrounding) environment is alien to the continuum. Therefore, the term "internal" as applied to energy also becomes superfluous, since the very concept "energy of a system" $\mathcal{E}$ already means that it belongs to the system as its "own", which belongs only to its value. However, in a continuous medium, there are no "field-forming" or "trial" bodies, to which "embellishment" is attributed. Consequently, to study the properties of primary matter, it is necessary to modify the law of Newton $F_{g}=G m M / R^{2}$ so that it corresponds to the continuum.

As is known, Newton's law was obtained under the assumption that the space between $m$ and $M$ is empty $(\rho=0)$, and the gravitational potential $\psi_{g}=-G M / R$ is negative and depends, besides the "field-forming" mass $M$, only from the distance $R$ to the center "trial" mass $m \ll M$, i.e. $\psi_{g}=\psi_{g}(M$, $R)$. Therefore, we express this potential in terms of the density of the medium $\rho$ in the nectar "trial" (unit) spherical volume $V_{c}$ with radius $R_{\mathrm{c}}$ :

$$
\psi_{g}(\rho)=-\left(G V_{c} / R_{\mathrm{c}}\right) \rho .
$$

Due to the constancy of the expression in parentheses, the acceleration of $g=-\nabla \psi_{g}$ can also be expressed as a function of the density gradient of the medium $\nabla \rho$ :

$$
\mathrm{g}=\left(G \rho V_{c} / R_{\mathrm{c}}\right) \nabla \rho / \rho=\psi_{g} \nabla \rho / \rho,
$$

It follows that a spontaneous violation of homogeneity in any region of the Universe $(\nabla \rho \neq 0)$ leads to the appearance of a pressure gradient $\nabla \rho$ of one or another sign $(\nabla g \neq 0)$ and generates a wave-formation process ("Sakharov oscillations") in the primary matter [10] in the widest frequency range $v$. The result of this process can be traced by the example of a single wave (Figure 1).

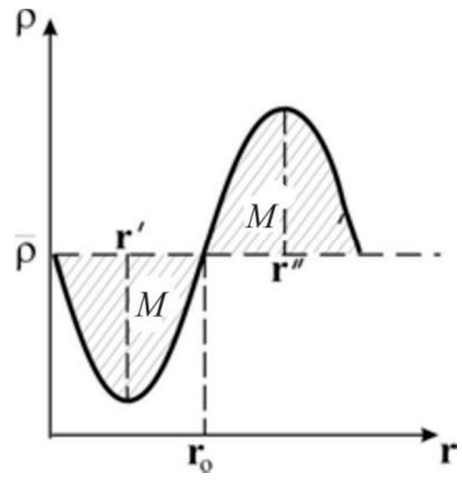

Figure 1. Wave formation in the primary matter.

It follows from it that any wave of an arbitrary extensive quantity в (in this case, the mass of the medium at rest $M$ with density $\rho$ ) is formed by transferring some of its quantity из 'from the position with the radius vector $r$ ' to the position $r$ ", i.e. by the half-wavelength $\lambda / 2$. The speed of this displacement $v$ varies from zero at the antinodes of the wave to the maximum at its nodes. Therefore, the process of wave formation in the primary matter is inextricably linked with overcoming inertia forces and performing work $d W^{k}=\mathrm{F}_{i} \cdot d \mathrm{r}$ $=v_{i} \cdot d \mathrm{P}$ against Newtonian forces inertia $\mathrm{F}=d \mathrm{P} / d t$, where $\mathrm{P}$ $=M_{o} v$ is the amount of motion (impulse) of the mass of primary matter $M$ involved in the oscillatory motion:

$$
\mathcal{E}^{k}=W^{k}=\int v_{i} \cdot d \mathrm{P}
$$

To calculate this work, we take into account that during the oscillation period $\tau$, the opposite of its frequency $v$, the reciprocating mass shift $M$ at a distance $\Delta \mathrm{r}$ is performed twice, so that the modulus of the average velocity of its displacement is equal to:

$$
\bar{v}=2|\Delta \mathrm{r}| / \tau=\lambda v .
$$

In this case, the work can be calculated, take this value is the same for oscillations of any frequency and equal to the speed of light in the "empty space" $\bar{v}=c=$ const: 


$$
\mathcal{E}^{k}=W^{k}=\int_{C} d M_{o} c=M c^{2} .
$$

Since in the antinodes of the wave $(v=0)$, this energy is completely converted into other forms of energy (for example, the energy of elastic deformation of primary matter), their sum remains unchanged at any time, equal to $\mathcal{E}^{\mathrm{k}}$. This determines the origin of the understanding of the energy of primary matter as a measure of its "hidden" motion $\mathcal{E}=M c^{2}$. This position is called the principle of equivalence of energy $\mathcal{E}$ and mass $M$ and is traditionally attributed to the special theory of relativity (STR). Meanwhile, their proportionality was established in relation to the ether long before its appearance (N. Umov, 1873; J. Thomson, 1881; O. Heaviside, 1990) [11]. Thus, the quantity $M c^{2}$, which we usually take for the total energy of the material system, is in its origin the energy of the oscillatory motion of the primary matter. Due to this energy, work is performed in the process of the emergence and structurization of the baryonic matter.

The density of this vibrational energy $\rho_{e}=\partial \mathcal{E} / \partial V$ is distributed unevenly in the volume of the wave and in the space of the Universe as a whole, which causes the appearance of a gravitational field with intensity

$$
\mathrm{H}_{e}=\nabla \rho_{e}=c^{2} \nabla \rho,\left(\mathrm{H} \mathrm{M}^{-3}\right)
$$

According to (8), any spontaneously arising fluctuation of the density of primary matter $\nabla \rho>0$ becomes the center of gravity of its surrounding mass, which leads to further unlimited local compaction of the primary matter. This is how the germs of all forms of the substance of the Universe arise, ranging from the nuclei of future atoms to the nuclei of metagalaxies. The work is done in this case $d W^{k}=\bar{v} d(M \bar{v})$ can be represented as two components:

$$
d W^{k}=\bar{v} d(M \bar{v})=\bar{v}^{2} d M+M d \bar{v}^{2}
$$

The first one is the work of mass input $M$ in the region of the formation of a baryon substance, and the second is the work expended on the process of structure formation in a baryon substance and on the excitation of oscillatory motion in it with a velocity $v$. This means that when $v<c$, the process of structure formation in a baryon substance is accompanied by the appearance of additional degrees of freedom (compared to the primary matter). Such are, in particular, internal thermal, chemical, electrical, magnetic and other forms of energy of a condensed substance. Each of them is distinguished by the specific nature of the movement and its impulse $\mathrm{P}_{i}=M_{i} \mathrm{v}_{i}$, determined by the mass $M_{i}$ and the matter involved in this movement and its speed $v_{i}$. This eliminates any arbitrariness in the interpretation of the physical meaning of the so-called "generalized coordinates" $\Theta_{i}$ and "generalized potentials" $\psi_{i}$ of various forms of energy. This opens up the prospect of a unification of the system of physical quantities and their units of measurement, which is extremely important both in terms of educational and methodological and metrological aspects. The difference in the vibrational spectrum of the structural elements of the baryonic substance from the background radiation of the primary matter makes this baryonic substance visible (observable). Understanding this circumstance determines the ability to return energy to the original meaning of the measure of motion.

\section{The Generalized Law of Conservation and Transformation of Energy}

It is known that energy does not just disappear in some points of space and appears in others, but is transferred by its material carrier (in short - energy carrier) across the system's borders. Based on these ideas, the Russian scientist N. Umov in 1873 proposed to write down the law of conservation of energy in the form [14]:

$$
d \mathcal{E} / d t=-\int \nabla \cdot \mathrm{j}_{e} d V
$$

where $\mathrm{j}_{e}$ is the energy flux through the vector element $\mathrm{df}$ of the closed surface of the system with volume $V$ in the direction of the outward normal $\mathrm{n}$ (Figure 2).

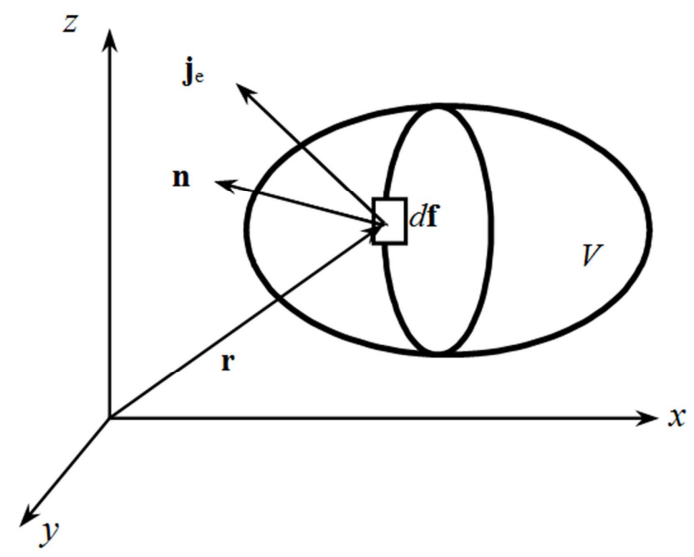

Figure 2. The flow of energy across system boundaries.

According to this expression, the loss of energy of a system is equal to the amount of energy that crosses its boundary like a liquid. In this form, the energy conservation law is the most general, since it takes into account the kinetics of real processes and the spatial heterogeneity of the object of study, without making any assumptions about its internal structure. The flow of energy $\mathrm{j}_{e}$ into any region of space with a volume $V$ may be due to different reasons: heat transfer, diffusion of $k$-th substances across the system's boundaries, charge transfer, etc. All such processes contribute to the energy flow which $\mathrm{j}_{e}$ can be expressed as the product of the energy flux density $\mathrm{j}_{i}$ by its potential $\psi_{i}$ [8]:

$$
\mathrm{j}_{e}=\Sigma_{i} \mathrm{j}_{e i}=\Sigma_{i} \psi_{i} \mathrm{j}_{i} \text {. }
$$

The universality of such a view becomes especially obvious due to the above interpretation of energy as a measure of motion. Then all the quantities $\mathrm{j}_{i}=\rho_{i} \mathrm{v}_{i}$, referred to in energy dynamics as the flux density of the $i$-th energy carrier $\Theta_{i}$ (entropy $S$, mass $M$, number of moles of $k$-th substances $N_{k}$, charge $Q_{e}$, etc.) acquire a single meaning the velocity $v_{i}$, is the meaning of the generalized potential $\psi_{i}$ (chemical $\mu_{k}$, electric $\varphi$, absolute temperature $T$ and pressure 
$p$, specific enthalpy $h$, etc.). In this case, after decomposing $\nabla \mathrm{j}_{e}=\Sigma_{i} \nabla\left(\psi_{\mathrm{i}} \mathrm{j}_{i}\right)$ into the terms $\Sigma_{i} \psi_{i} \nabla \mathrm{j}_{i}+\Sigma_{i} \mathrm{j}_{i} \nabla \psi_{i}$ the energy conservation law (8) takes the form [15]:

$$
d \mathcal{E} / d t=-\Sigma_{i} \int \psi_{i} \nabla \cdot \mathrm{j}_{i} d V-\Sigma_{i} \int \mathrm{j}_{i} \cdot \nabla \psi_{i} d V
$$

If we now take out the integral value for some average value $\Psi_{i}$ of the local potential $\psi_{i}$ and the average value $X_{i}$ of the potential gradient $\nabla \psi_{i}$, the energy conservation law can be expressed by the parameters of the nonequilibrium system as a whole, as is customary in classical thermodynamics:

$$
d \mathcal{E} / d t=\Sigma_{i} \Psi_{i} d \Theta_{i} / d t+\Sigma_{i} \mathrm{X}_{i} \cdot \mathrm{J}_{i},
$$

where $\mathrm{J}_{i}=\int \mathrm{j}_{i} d V=\Theta_{i}$ is the total flow (impulse) of the energy carrier $\Theta_{i}$.

In homogeneous (internally equilibrium) systems, where $\mathcal{E}$ $=U ; \mathrm{X}_{i}=\nabla \psi_{i}=0$ и $\Psi_{i}=\psi_{i}$, this equation takes the form:

$$
d U=\Sigma_{i} \psi_{i} d \Theta_{i}
$$

A special case of this expression for open thermomechanical systems is the classical Gibbs relation [2]

$$
d U=T d S-p d V+\Sigma_{k} \mu_{k} d N_{k}
$$

where $S, V, N_{k}$ - entropy, volume and number of moles of the $k$-th substance; $T, p, \mu_{k}$ - its absolute temperature, pressure and chemical (or electrochemical) potential.

In non-equilibrium systems, where the parameters $S, V, N_{k}$ can change spontaneously, this equation becomes inequality, which gives rise to the well-known problem of thermodynamic inequalities [1]. This circumstance for a long time prevented the application of classical thermodynamic methods to non-equilibrium systems. Meanwhile, the equal sign in the relation (15) can be preserved if, according to (12), the potentials $T, p, \mu_{k}$ are replaced by their mean values $\bar{T}, \bar{p}$ и $\bar{\mu}_{k}[16]$.

It is also easy to notice that when potentials $\psi_{i}$ are replaced by their average integral value $\Psi_{i}$, the first sum (13) in isolated systems vanishes due to the lack of transfer of any energy carriers across the system borders $\left(d \Theta_{i} / d t=0\right)$. In this case, $d \mathcal{E} / d t=0$, and equation (12) goes into the law of conservation of energy during its interconversions:

$$
\Sigma_{i} \mathrm{X}_{i} \cdot \mathrm{J}_{i}=0 .
$$

Thus, equation (12) includes the law of conservation of energy during its transformations in non-equilibrium systems. It is this form of the conservation law that is used in energy dynamics [8], which, due to this, can be considered as the thermodynamics of inhomogeneous isolated systems. It makes it possible to obtain a series of non-trivial consequences concerning the very concept of energy $\mathcal{E}$.

\section{Energy as the Most Common Function of the System State}

The basic equation of energy dynamics in the form of (12) indicates the failure of the local equilibrium hypothesis [16], according to which elements of a nonequilibrium continuum are characterized by the same set of variables as in equilibrium. To do this, it suffices to point out the presence of potential gradients in them, which determine the thermodynamic force $\mathrm{X}_{i}$, which is the most important for thermodynamics of irreversible processes (TIP) [17]. To find out the meaning of additional degrees of freedom of nonequilibrium systems, it is sufficient to compare the center of $\mathrm{r}_{i}$ with any extensive parameter $\Theta_{i}$ (mass $M$, numbers of moles $k$-th of substances or phases $N_{\mathrm{k}}$, entropy $S$, charge $\Theta_{e}$, impulse components $\mathrm{P}$, its momentum $\mathrm{L}$, etc. ) in the current and equilibrium rio state (with energy density $\bar{\rho}_{i}=\Theta_{i} / V$. This position is determined by the well-known expressions:

$$
\mathrm{r}_{i}=\Theta_{i}^{-1} \int \rho_{\mathrm{i}} \mathrm{r} d V ; \mathrm{r}_{i o}=\Theta_{\mathrm{i}}^{-1} \int \bar{\rho}_{i} \mathrm{r} d V
$$

This immediately implies the emergence of a certain "distribution moment" $\mathrm{Z}_{i}=\Theta_{i}\left(\mathrm{r}_{i}-\mathrm{r}_{i \mathrm{o}}\right)$, which characterizes the inhomogeneous state of the system as a whole. These extensive parameters are conjugate to the potential gradients $\nabla \psi_{i}$, just as in equilibrium thermodynamics, the variables $\Theta_{i}$ are conjugated to the potentials $i$. It is easy to see that the time derivatives of these moments determine the so-called fluxes $\mathrm{J}_{i}$, with which thermodynamics of irreversible processes operate [17]:

$$
\mathrm{J}_{i}=d \mathrm{Z}_{i} / d t=\Theta_{\mathrm{i}} d \mathrm{r}_{\mathrm{i}} / d t=\Theta_{i} \mathrm{v}_{i} .
$$

As you can see, the non-uniform distribution of any energy source $\Theta_{i}$ is characterized by the "displacement vector" of its center $\Delta \mathrm{r}_{i}=\mathrm{r}_{i}-\mathrm{r}_{i \mathrm{o}}$. This means that the energy $\mathcal{E}_{i}$ of each $\mathrm{i}$-th degree of freedom of a nonequilibrium system as a function of its state is characterized by at least twice (compared to equilibrium) number of arguments $\mathcal{E}_{i}=\mathcal{E}_{i}\left(\Theta_{i}, \mathrm{r}_{i}\right)$, and the total differential of the system as a whole $\mathcal{E}=\Sigma_{i} \varepsilon_{i}$ can be represented in the form of an identity [8]:

$$
d \mathcal{E} \equiv \Sigma_{i} \Psi_{i} d \Theta_{i}+\Sigma_{i} \mathrm{X}_{i} d Z_{i},
$$

where $\Psi_{i} \equiv\left(\partial \mathcal{E} / \partial \Theta_{i}\right)$ is the averaged value of the generalized potential (absolute temperature $T$ and pressure $p$, chemical $\mu_{k}$, electric $\varphi$, gravitational $\varphi_{g}$, and other potentials); $\mathrm{X}_{i} \equiv$ $\left(\partial \mathcal{E} / \partial \mathrm{Z}_{i}\right)$ is a measure of the inhomogeneity (intensity) of the system state. The connection of $\mathrm{X}_{i}$ with the force $\mathrm{F}_{i}$ is easy to establish, considering that the derivative $\left(\partial \mathcal{E} / \partial Z_{i}\right)$ is in the condition of constancy $\Theta_{i}$. Then $\mathrm{X}_{i}=\left(\partial \mathcal{E} / \partial \mathrm{Z}_{i}\right)=\mathrm{F}_{i} / \Theta_{i}=\Theta_{i}^{-}$ ${ }^{1}\left(\partial \mathcal{E} / \partial \mathrm{r}_{i}\right)$, that is, it has the meaning of the specific internal force.

It is easy to see that identity (19) is adequate to equation (12), which in this case becomes a direct consequence of the dependence of the energy of a nonequilibrium system $\mathcal{E}$ on the nonequilibrium parameters of the system $X_{i}$ and $Z_{i}$. Due to this, both expressions, (13) and (19), remain valid for any values of its arguments and for any (reversible or irreversible) nature of the processes. This circumstance eliminates the above-mentioned problem of thermodynamic inequalities [17], allowing the thermodynamic method to be distributed to systems far from equilibrium

One of the most important consequences of identity (19) is 
the possibility of the emergence at each $i$-th form of the partial energy $\mathcal{E}_{i}$ of new degrees of freedom corresponding to the further decomposition of the parameters $Z_{i}=\Theta_{i} \Delta r_{i}$ into independent components. This circumstance is directly related to the concept of the number of degrees of freedom of a nonequilibrium system, by which we mean the number of arguments necessary and sufficient to uniquely determine its energy.

To find out this number, we take into account that the displacement velocity $v_{i}=d \mathrm{Z}_{i} / d t$ can be decomposed into translational $\mathrm{w}_{i}$ and rotational $\left[\omega_{i}, \mathrm{r}_{i o}\right]$ components:

$$
\mathrm{v}_{i}=\mathrm{w}_{i}+\left[\omega_{i}, \mathrm{r}_{i o}\right],
$$

where $r_{i o}$ is the instantaneous radius of rotation of the body or particle).

Accordingly, one more sum $\Sigma_{\mathrm{i}} \mathrm{M}_{\mathrm{i}} \mathrm{d} \varphi_{\mathrm{i}}$ will appear in identity (19), where $\varphi_{i}$ is the spatial angle of the vector $r_{i}$, whose derivative with respect to time determines the angular velocity $\omega_{i}=d \varphi_{i} / d t$ of its rotation under the action of the torque $\mathrm{M}_{i}=\partial \mathcal{E} / \partial \varphi_{i}$.

If in a baryon substance there is a translational and rotational oscillatory motion of energy carriers $\Theta_{i}$ with a frequency $v$, then the system under consideration acquires additional degrees of freedom, whose energy is determined by the sum $\mathcal{E}_{i}^{k}=M_{i} \bar{w}_{i}{ }^{2} / 2+I_{\omega} \bar{\omega}_{i}{ }^{2} / 2$, where $\bar{w}_{i}, \bar{\omega}_{i}$ - linear and angular averaged over the period the speed of oscillatory motion, $I_{\omega}$ is the moment of inertia. In this case, the number of arguments $\mathcal{E}_{i}$ will include additional scalar pulses $P_{w i}=M_{i}$ $\bar{w}_{i}$ and moments $L_{\omega i}=I_{\omega} \bar{\omega}_{i}$ of the internal oscillatory motion. In this case, identity (19) takes the form:

$$
d \mathcal{E} \equiv \Sigma_{i} \Psi_{i} d \Theta_{i}+\Sigma_{i} \mathrm{~F}_{i} \cdot d \mathrm{r}_{i}+\Sigma_{i} \mathrm{M}_{i i} \cdot d \varphi_{i}+\Sigma_{i} \bar{w}_{i} d P_{w}+\Sigma_{i} \bar{\omega}_{i} d L_{\omega i} \cdot
$$

Thus, the energy of the system $\mathcal{E}$ as a function of state has in the general case the form $\mathcal{E}=\Sigma_{i} \mathcal{E}_{i}\left(\Theta_{i}, \mathrm{r}_{i}, \varphi_{i}, P_{w i}, P_{\omega i}\right)$. If we take into account that the variables $r_{i}$ and $\varphi_{i}$ have three independent Cartesian components, and the same $i$-th form of energy $\mathcal{E}_{i}$ is inherent in each $k$-th independent component of the system in any of the $\gamma$ its independent phases, it becomes clear that the number of independent energy arguments $\mathcal{E}$ condensed matter, i.e. the number of its degrees of freedom, can reach very significant, albeit finite, values. Since not enough attention is paid to the analysis of this issue in educational literature, a very common methodological mistake of modern theories was an attempt to describe the state of the system with a missing or redundant number of variables (i.e., "underdetermination" or "redefinition" of the system). A typical example of this is the already mentioned local equilibrium hypothesis. From this, it follows that when constructing any system of energy classification, it should be understood as the most general function of the system state, the number of arguments being equal to the number of independent processes occurring in the system under study. Such an understanding of energy is very close to its definition by Maxwell as "the sum of all the actions that a system can produce on surrounding bodies" [19].

\section{The Laws of Conservation in the Transfer and Transformation of Energy}

Equations (13) and (19) divide the energy exchange processes into two groups, which differ in both physical meaning and tensor rank. In order to understand the physical meaning of their differences, let us pay attention to the fact that, according to (13), the members of its first sum characterize changes in energy caused solely by the transfer of energy carrier $\Theta_{i}$ across the system boundaries. Such are the processes of heat transfer, volumetric deformation (exchange of occupied space), diffusion of $k$-x substances across system boundaries, etc. This means that members of their 1 st sum

$$
d \mathcal{E}=\Sigma_{i} \Psi_{i} d \Theta_{i}
$$

characterize the processes of energy transfer (transit) in the same form, corresponding to the nature of the energy carrier $\Theta i$. Such processes in energodynamics are called energy transfer processes [8]. At the same time, elementary heat exchange $d Q=T d S$, the extension work $d W_{p}=p d V$ and the input work of the $k$ th substance $d W_{k}=\mu_{k} d N_{k}$ are in the same process group. The essence of the matter is that all these processes are not connected with overcoming the resultant of any forces. This can be verified using the example of a comprehensive expansion, taking into account that, according to the gradient theorem, the integral of the product of pressure $p$ on the element $d f$ of a closed system surface, which determines the resulting pressure force $\mathrm{F}_{p}$ is zero if there are no pressure gradients $\nabla p$ in it:

$$
\mathrm{F}_{p}=\int p d f=\int \nabla p d V=0 .
$$

This kind of work $d W_{i}^{H}$ which is not associated with a change in the position of the center of mass of the body is called disordered in energy dynamics. It serves as a quantitative measure of energy transfer, i.e. exchange between parts of the system (subsystems) of energy in the same $i$-th form. The refusal of energy dynamics to divide energy exchange into heat exchange and work has a deep meaning. The point is not only that in open systems such a division is impossible due to the presence of diffusion, which cannot be reduced either to heat or to work [20]. The heat exchange $d Q$ is essentially in the transfer of the impulse of particles of one body to another and therefore is also associated with overcoming their inertia forces, that is, represents a kind of "microwork" of acceleration. This means that, from the phenomenological point of view, entropy should not be interpreted as a measure of chaos, but as a "thermoimpulse", that is, an impulse of particles of matter that has lost its vector nature due to the randomness of the thermal motion [8]. Naturally, the thermoimpulse can change both as a result of heat exchange $d Q$ and as a result of the occurrence of internal heat sources dissipation $Q^{d}$, which directly leads to the entropy balance equation [15]: 


$$
T d S=d Q+d Q^{d} .
$$

This removes the cover of mystery from both entropy and the principle of its growth.

Another kind of work described by members of the 2 nd sum (13) and (19):

$$
d W_{i}^{\mathrm{T}}=\mathrm{F}_{i} \cdot d \mathrm{R}_{i}=\mathrm{X}_{i} \cdot d \mathrm{Z}_{i}
$$

It is this kind of $d W_{i}^{\mathrm{T}}$ work featured in mechanics and other disciplines that operate on the concept of force $F_{i}$. Thermodynamics of irreversible processes, which operates with the notion of the thermodynamic force $\mathrm{X}_{i}$ [21], does not constitute an exception. This kind of work is, as is known, a quantitative measure of the process of energy conversion (transformation of energy from one form to another) $W^{\mathrm{T}}$. In equilibrium thermodynamics, where the concept of force $\mathrm{F}_{i}$ or $\mathrm{X}_{i}$ is absent, this kind of work is described by the same variables $\psi \mathrm{i}$ and $\Theta_{i}$, which creates unnecessary difficulties in understanding the difference between these types of work. Such is, in particular, the work of gas in the stream $d W_{p}^{\mathrm{T}}=$ $V d p$ [22], which different authors call technical, useful external, available, etc. Their fundamental difference from the work of the extension $d W_{p}^{\mathrm{H}}=p d V$ is the directional nature of moving the object of application of force Fi. Since not only technical, but also biological, ecological, cosmological, etc. systems perform such work, and the work itself can be not only external, but also internal, and not only useful but also dissipative, in energodynamics, such work is called just ordered [23]. Understanding that the "work of work is different" is facilitated by taking into account the tensor rank of parameters that appear in their analytical expressions. It plays a crucial role in the interpretation of energy as the ability of the system to perform any (external and internal, ordered or unordered, useful and dissipative) work.

\section{Partial Energies and the Principle of Their Additivity}

In the study of polyvariant systems (with many degrees of freedom), it is completely insufficient to divide the energy into kinetic and potential. The fact is that the number of components $\mathcal{E}_{i}$ of the system energy in the general case is much more than the known forms of its kinetic and potential components. For example, the concept of potential deformation energy includes linear, shear and volume deformation, torsion deformation, elastic and plastic deformation, etc. The kinetic energy of any component of the system corresponds to independent forms of translational, rotational and oscillatory motion. The latter, in turn, is different for its independent modes (frequencies and phases) of oscillations. Even more difficult is the chemical energy of substances, which corresponds to the set $k=1,2, \ldots, K$ of the energies of the components of the mixture $\Sigma_{k} \mu_{k} N_{k}$. In all such cases, it is necessary to decompose energy into independent components. This makes it expedient to introduce the concept of "partial" energy $\mathcal{E}_{i}$ (from Latin partialis partial) as energy corresponding to independent energy carrier $\Theta_{i}$. To this end, we turn to equation (10), according to which the energy carrier flux density $j_{i}$ is expressed as the product of the density $\rho_{i}=\partial \Theta_{i} / \partial V$ of its carrier $\Theta_{i}$ by the transfer rate of the latter vi. Then $j_{e}=\rho_{e} v_{i}=\Sigma_{i} \psi_{i} \rho_{i} v_{i}$, where and $\rho_{e}=\partial \mathcal{E} / \partial V$. It follows that

$$
\mathcal{E}=\Sigma_{i} \mathcal{E}_{i}=\Sigma_{i} \int \psi_{i} \rho_{i} d V .
$$

Equation (26) expresses the principle of additivity of partial energies, according to which the energy of a polyvariant system is equal to the sum of its partial energies $\mathcal{E}_{i}=\int \psi_{i} \rho_{i} d V$ [24]. The partial energy $\mathcal{E}_{i}$ of any form of motion is a purely positive quantity, since motion, unlike its speed, cannot be negative.

The introduction of the concept of partial energy complements the very fruitful method of characteristic functions used in classical thermodynamics. At the same time revealing the physical meaning of these functions. One of the most frequently used functions are the "Helmholtz free energy $\Gamma=U-T S$, which is the difference between the internal energy and the component $T S$, referred to as "bound energy" [1]. From the standpoint of energy dynamics, this component has the meaning of the partial thermal energy of the system $\mathcal{E}_{q}=T S\left(\psi_{i}=T, \Theta_{i}=S\right)$. In this case, the "free" energy of Helmholtz acquires the meaning of the non-thermal ("non-entropic") component of the internal energy. Similarly, the component $p V$ of the Gibbs free energy $G=U+p V-T S$, becomes the meaning of the partial energy of elastic deformation $U_{p}=p V$. It is part of the internal potential energy $U_{\mathrm{n}}$, which participates in thermodynamic processes, in contrast to the "zero" energy $U_{\mathrm{o}}=U_{\text {п }}-p V$, which does not participate in them. In this case, the Gibbs free energy acquires the meaning of the remainder term minus the thermal and potential energy, which justifies its representation through the partial chemical energies of the $\mathcal{E}_{\mathrm{x}}$ $=\Sigma_{k} \mu_{k} N_{k}$ components of the system [24]. Thus, the introduction of the concept of partial energy and their representation by the product of the intensive and extensive parameters of the state allows us to give a more detailed and at the same time more "physical" classification of energy.

\section{Anergy as an Irreducible Part of an Energy}

In the thermodynamic literature, the division of all forms of energy into "non-entropic" (fully convertible) and "entropic" (incompletely transformable) is often found [25]. At the same time, the mechanical, electromagnetic and nuclear forms of energy (although it can be converted only to the extent of a mass defect) are attributed to fully convertible, and thermal and chemical energy to an incompletely convertible form. The inconsistency of such a representation follows directly from the identity (19), according to which any form of energy is convertible to the extent that it is nonequilibrium. Indeed, if the system is internally balanced 
(spatially homogeneous), then all the forces in it are $\mathrm{X}_{i}=0$, and, according to (19), the interconversion of various forms of energy in it is excluded. It also follows from this identity that in the composition of any form of partial energy $\varepsilon_{i}$, we can distinguish its equilibrium (irreversible) and the nonequilibrium (transformable) part corresponding to the first and second sum of the identity (19). To find the equilibrium component of the partial energy, it suffices in the expression (26) to take the average value $\Psi_{i}$ of the potential $\psi_{i}$ beyond the integral sign:

$$
\overline{\mathcal{E}}_{i}=\Sigma_{i} \Psi_{i} \Theta_{i}
$$

This irreducible part of the energy should be called anergy $^{2)}$ [26]. This part of the energy is only capable of transferring e across the system's boundaries in the process of performing the disordered operation of $W_{i}^{\mathrm{H}}$, as this takes place in the processes of heat exchange and mass transfer.

Rest of the energy

$$
\tilde{\mathcal{E}}_{i}=\mathcal{E}_{i}-\Psi_{i} \Theta_{i}
$$

represents its non-equilibrium (convertible) part, capable of accomplishing an ordered inner work. It differs fundamentally from the concept of exergy as a technically suitable part of energy [27] in that it does not depend on environmental parameters and their oscillations [28] and is a parameter of the state of the non-equilibrium (expanded) system. Such a non-equilibrium (convertible) part exists in principle for each (including thermal) energy. This circumstance was well understood by S.Carno (1824) when he wrote about the occurrence of the driving force of heat in the presence of caloric temperature difference [1]. Indeed, if by $\mathcal{E}_{i}$ we understand thermal energy and denote by $\Psi_{i}{ }^{\prime}$ and $\Psi_{i}{ }^{\prime \prime}$ the average temperatures of heat supply and removal in the cycle of a heat engine, then its thermal efficiency

$$
\eta_{\mathrm{t}}=1-\Psi_{\mathrm{i}}^{\prime \prime} / \Psi_{\mathrm{i}}^{\prime}
$$

the meaning of the degree of convertibility of thermal energy as a function of the thermal inhomogeneity of the system. This expression does not depend on the form of partial energy, which indicates the unity of the laws of transformation of any form of energy in cyclic machines.

In itself, the fact that energy as a measure of motion is not fully transformed into its various forms may seem contradictory to the concept of kinetic energy. However, one should not forget that this is not about external kinetic energy, measured by the amount of ordered work, but about the internal movement of a part of internal energy. Being scattered (lost vector nature) this movement loses the ability to transform completely. Therefore, such a movement is not accidentally called "hidden".

The introduction of the concept of disordered work facilitates the understanding of the difference between the

\footnotetext{
2) The term "anergy" to designate technically unsuitable (inoperable) part of the energy of the expanded system was proposed by Z. Rant [27].
}

concepts of "transformation" and "health" of energy. In this regard, the concept of anergy of any form of partial energy as its irreducible part is more informative than the concept of "internal potential energy". The latter implies the possibility of performing orderly work in the event of motion and energy conversion. Anergy as a measure of the equilibrium part of the "hidden" movement excludes this possibility. Thus, it turns out that the true "dividing line" in relation to transformation is not between the kinetic and potential energy, but between its equilibrium and non-equilibrium parts.

This circumstance must be borne in mind in connection with the endless discussions about the possibility of creating "super-unit" energy generators, the output power of which exceeds the amenable power at its input. There comes an understanding that the source of this excess capacity is the primary matter, having a non-uniform velocity field of oscillatory motion [28]. That is what makes it an inexhaustible source of energy for stars and planets, many orders of magnitude higher than the energy of thermonuclear fusion. Therefore, the study of the processes of accretion of primary matter and its condensation offers promising prospects for the transition to non-fuel energetics.

\section{Conclusion}

1. The existing uncertainty of the concept of energy was the result of the unreasonable expansion of the original meaning of this concept as a measure of movement by introducing the concepts of potential energy, not related to movement, and internal energy, which is not a measure of efficiency. In non-equilibrium and closed systems, internal energy $\mathcal{E}$ ceased to be "scattered", and external energy became part of it. The result of the free treatment of this concept was the situation when modern physics no longer know what energy is.

2. The recognition of the presence in the primary matter of latent forms of oscillatory motion gives its potential energy a sense of the measure of the heterogeneity of this motion. This meaning is also preserved in all forms of the potential energy of a baryon substance formed in the process of its condensation. This allows us to return the concept of energy to the original meaning of the most general measure of motion, eliminating from its definition a very vague concept of interaction. This approach leads to the interpretation of all forms of kinetic energy as functions of the amount of motion of the corresponding kind and all forms of potential energy as functions of its distribution. Its implementation promises a significant simplification of the conceptual and metrological system of natural science disciplines.

3. Another way is to use the kinetic form of the law of conservation of energy, in which its change in time is associated with the flow of energy and energy through the system's boundaries. This path leads to the solution of the problem of thermodynamic inequalities, to the 
distinction between ordered and disordered work, energy transfer and energy conversion, to the refusal to divide energy exchange into heat exchange and work, to the release of its ordered and disordered part (inergy and energy), etc. This way allows you to return the energy close to the original meaning of the measure of system performance.

4. The third way is to represent the energy of the system as the most common characteristic function of the state, whose derivatives allow, by its arguments, to find the potentials and driving forces of all independent processes occurring in it. This path reveals the inconsistency of the hypothesis of local equilibrium and confirms the need to introduce the missing extensive parameters of nonequilibrium, coupled with potential gradients. It leads to the law of conservation of energy in the form of an identity, which does not exclude from consideration any (reversible or irreversible) part of real processes. This allows you to extend the thermodynamic method of research to other fundamental disciplines.

5. All these paths lead to an understanding of the energy of a system as the most general function of its state, the number of arguments of which is equal to the number of processes occurring in the system. The internal (self) energy of a system is a measure of all forms of its internal movement and characterizes the ability of the system to act, that is, to perform any (orderly and disordered) work. Energy is an absolute value invariant with respect to reference systems, and all of its components (partial energies) are purely positive values, including a transformable and irreducible part (inergy and anergy).

6. Such an understanding of energy allows us to distinguish it from any other conserved value and makes it unnecessary to formally divide it into external and internal, free and connected, entropic and nonentropic, positive and negative due to the ambiguity of their meaning in real (irreversible) processes. The proposed definition of energy and its classification eliminates the existing uncertainty of the concept of energy and opens up the possibility of creating a unified system of dimensions of quantitative and qualitative measures of all its forms.

7. All this gives the "own" energy of the system the meaning of the most general function of its state, serving as a single measure of all forms of its movement and characterizing its ability to perform any work (external and internal, ordered and disordered, useful and dissipative). The number of arguments of this function is equal to the number of macroprocesses occurring in the system, and all its components (according to the number of arguments) are purely positive values that are independent of external reference systems. Such a definition of energy is acceptable for any natural science discipline and is close to its original meaning of the measure of "living force".

\section{References}

[1] Gelfer J. M. History and Methodology of Thermodynamics and Statistical Physics. Ed. 2. - M: Vysshaya shkola, 1981 (In Russian).

[2] Bazarov I. P. Thermodynamics. Edn 4. M.: Vysshaya shkola, 1991 (in Russian).

[3] Feynman R. Character of Physical Laws.- M.: Physical Encyclopedia, 1984. (In Russian).

[4] Landau L. D., Livshits E. M. Theoretical physics. T. 1. Mechanics.- M.: Science, 1973 (In Russian).

[5] Poincaré H. On Science.-M.: Nauka, 1983. (In Russian).

[6] Physical encyclopedic dictionary. - M.: "Soviet Encyclopedia", 1984. (In Russian).

[7] Feynman R., Leyton R., Sands M. Feynman's Lectures on Physics. V. 5, 6. - London, 1964.

[8] Etkin V. Energodynamics (Thermodynamic Fundamentals of Synergetics).- New York, 2011.- 480 p.

[9] Ade P. A. R. et al. Planck 2013 results. I. Overview of products and scientific results. //Astronomy and Astrophysics, 1303: 5062

[10] Sakharov A. D. The initial stage of the expansion of the Universe and the occurrence of inhomogeneity in the distribution of matter // Journal of Experimental and Theoretical Physics, 49 (1965). 345 (in Russian).

[11] Whittaker E. A History of the Theories of Aether and Electricity. The Modern Theories 1900-1926, London: Thomos Nelson, 1953.

[12] Eisenstein, D. J.; et al. Detection of the Baryon Acoustic Peak in the Large-Scale Correlation Function of SDSS Luminous Red Galaxies. //The Astrophysical Journal, 633 (2). 2005. 560.

[13] Etkin VA. On Wave Nature of Matter. // World Scientific News 69, (2017). 220-235.

[14] Umov A. I. Selected Works. M. L., 1950. p. 203.

[15] Etkin V. A. The fundamental equation of non-equilibrium thermodynamics.// Russian Journal of Physical Chemistry, 62 (8), 1988.1157-1159 (translated from Zhurnal Fizicheskoi Khimii, 62 (1988). 2246-2249.

[16] Prigogine I. Time, structure and fluctuations (Nobel lecture in chemistry in 1977). // Successes of physical sciences, 131 (1980).185... 207. (In Russian).

[17] Demirel Y. Nonequilibrium Thermodynamics. Transport and Rate Processes in Physical, Chemical and Biological Systems, 3rd ed., Amsterdam, 2014.

[18] Etkin V. A. To a solution of the problem of thermodynamic inequalities. // Soviet. Journal of Appl. Physics, 1988, 4 (15), pp. 274-279 (translated from Bulletin of REssian Acad. of Science, Siberian Branch.- Engineering, 1988.- 4 (15), pp. 3439).

[19] Maxwell J. The Treatise on Electricity and Magnetism. T. 1., M., Nauka, 1989. 
[20] Tribus M. Thermostatics and Thermodynamics. M.: Energiya, 1970. (In Russian).

[21] De Groot S. R., Mazur R. Non-Equilibrium Thermodynamics:- Amsterdam, 1962.

[22] Andryushchenko A. I. Fundamentals of technical thermodynamics of real processes. M.: Higher. School, 1975. (In Russian).

[23] Etkin V. A. Heat and work in irreversible processes. //Izv. universities. Energetics, 4 (1988). 118-122. (In Russian).

[24] Etkin V. A. Full and partial energy of the system. // Bulletin of the Sc. House of Haifa, 36 (2016). 6-11 (In Russian).
[25] Exergy calculations of technical systems. // Handbook ed. A. A. Dolinsky and V. M. Brodyansky, Kiev, 1991.

[26] Etkin V. A. Energy and anergy. // Bulletin of the Sc. House of Haifa, 9 (2006). 30-38 (In Russian).

[27] Rant, Z. Exergie, ein neues Wort fur «technische Arbeitsfähigkeit». Forschung auf dem Gebiet des Ingenieurwesens, 22 (1), 1956. 36-37.

[28] Etkin V. A. Theoretical basis of fuel-free energy. Saarbrǘken, 2013. 